\title{
Multi-walled carbon nanotube filled polypropylene nanocomposites based on masterbatch route: Improvement of dispersion and mechanical properties through PP-g-MA addition
}

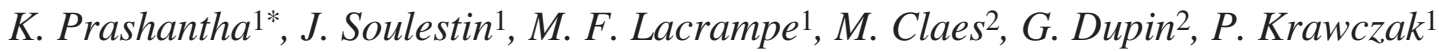 \\ ${ }^{1}$ Ecole des Mines de Douai Polymers and Composites Technology \& Mechanical Engineering Department, \\ 941 rue Charles Bourseul, BP 10838, 59508 Douai Cedex, France \\ ${ }^{2}$ Nanocyl S.A. Rue de l'Essor, 4 B-5060 Sambreville, Belgium
}

Received 18 July 2008; accepted in revised form 14 September 2008

\begin{abstract}
Multi-wall carbon nanotubes (MWNTs) filled polypropylene (PP) nanocomposites were prepared through diluting a PP/MWNT masterbatch in a PP matrix by melt compounding with a twin screw extruder. Polypropylene grafted maleic anhydride (PP-g-MA) was used to promote the carbon nanotubes dispersion. The effect of PP-g-MA addition on the rheological, mechanical and morphological properties of the nanocomposites was assessed for different MWNTs loadings. Scanning electron microscopy (SEM) has shown that nanotubes are distributed reasonably uniformly. A better dispersion and good adhesion between the nanotubes and the PP matrix is caused by wrapping of PP-g-MA on MWNTs. When PP-gMA is added, dynamic moduli and viscosity further increases compared to PP/MWNT nanocomposites. The rheological percolation threshold drops significantly. Tensile and flexural moduli and Charpy impact resistance of the nanocomposites also increases by the addition of PP-g-MA. The present study confirms that PP-g-MA is efficient to promote the dispersion of MWNTs in PP matrix and serves as an adhesive to increase their interfacial strength, hence greatly improving the rheological percolation threshold and mechanical properties of PP/MWNT nanocomposites.
\end{abstract}

Keywords: nanocomposites, multi wall carbon nanotubes, polypropylene, masterbatch, PP-g-MA

\section{Introduction}

Carbon nanotubes (CNTs) are ideal fillers for polymer composites due to their high Young's modulus combined with good electrical and thermal conductivity. The very high aspect ratio makes it likely that the addition of a small amount of CNTs strongly improves the electrical [1], thermal [2] and mechanical [3] properties of the polymer matrix. Thus, CNT/polymer composites combine the good processability of the polymers with the excellent mechanical and other functional properties of the CNTs. However, the strong intermolecular van der
Waals interactions among the nanotubes, in combination with their high surface area and high aspect ratio, commonly causes significant agglomeration, and prevents transfer of their superior properties to the matrix [4]. Thus, dispersion of CNTs in polymer matrices plays a predominant role in the mechanical and other functional properties of polymer/CNT composites [5].

To disperse CNTs into the polymers homogeneously, the entanglement of CNTs inherited from their synthetic process and van der Waals forces between CNTs should be minimized. Good disper-

*Corresponding author, e-mail: prashantha@ensm-douai.fr

(C) BME-PT and GTE 
sion has been reported for polar polymers such as poly(methyl methacrylate) [6], polycarbonate [7], polyacrylonitrile [8] and poly(vinyl alcohol) [9]. However, CNT dispersion in non-polar polymers such as polypropylene during melt processing remains a challenge. Techniques such as end-group functionalization [10-12], use of ionic surfactants [13], shear mixing [14, 15] and plasma coating [16] have been used to improve dispersion and exfoliation of nanotubes in polypropylene matrix. Polypropylene compatibility with fillers has been improved by matrix modification by grafting it with reactive moieties, such as acrylic acid, acrylic esters, and maleic anhydride $[17,18]$. In that case, improvement in thermal and electrical properties of polypropylene/nanotube composites has been reported [14, 19-21].

Compatibilizers containing maleic anhydride functionalities are effective in improving physical properties of composites. The interaction between functional groups of the compatibilizer and carboxyl or amine groups of multi wall carbon nanotubes (MWNTs) has stabilized the morphology and improved the interfacial interaction between MWNTs and the polypropylene matrix [22]. Especially maleic anhydride grafted polypropylene (PPg-MA) is one of the most promising candidates that can improve the PP/MWNT composite properties. In general, the nanoscale dispersion of MWNTs in polypropylene matrix is achieved by strong hydrogen bonding between hydroxyl groups of the MWNTs and maleic anhydride groups of PP-gMA, depending upon the chemical similarity of polypropylene matrix and the grafted polypropylene [22].

Another key issue is related to handling of CNTs in plastic parts manufacturing workshop. Using commercial masterbatch in the production of CNTs based polymer nanocomposites would be a better choice as it offers a dust free solution, with no health and safety risks in comparison to bulk nanotube dispersion process. Other benefits of using masterbatch include elimination of dispersion difficulties, formulation development and easy handling. Nevertheless, distribution of masterbatch and subsequent dispersion of nanotubes in the polymer matrix after processing needs to be ascertained. Recent studies from our laboratory reported the compounding of masterbatch based PP- multi wall carbon nanotubes (MWNTs) nanocomposites using twin screw extrusion process [23]. The results showed good mechanical properties, similar to those obtained with direct mixing of MWNTs in PP matrix as reported in the literature [24, 25]. However, scanning electron microscopy (SEM) observations revealed a few masterbatch aggregates which were imperfectly mixed during extrusion.

Therefore, in the present work, PP-g-MA is used to enhance the dispersion of MWNTs in masterbatch based PP/MWNT nanocomposites. The mechanism promoting the improved dispersion induced by the PP-g-MA will be discussed through a microscopic study. The influence of the PP-g-MA addition on structural and mechanical properties of PP/MWNT composites will be discussed in conjunction with the microscopic studies.

\section{Experimental}

\subsection{Composites fabrication}

Polypropylene (PP)-multi wall carbon nanotubes (MWNTs) nanocomposites were produced by mixing homo PP granules [Polychim polypropylene with a melt flow index of $12 \mathrm{~g} / 10 \mathrm{~min}$. at $190^{\circ} \mathrm{C}$ ] and 2 wt $\%$ PP-g-MA [Dupont Fusabond] with the commercial masterbatch containing $20 \mathrm{wt} \%$ of MWNT ['Plasticyl 2001' supplied by Nanocyl, Belgium] in a co-rotating twin screw extruder [Clextral BC] at barrel temperature of $195-210^{\circ} \mathrm{C}$, and a screw speed of $50 \mathrm{rpm}$. The specifications of MWNTs in the masterbatch are as follows: range of diameter 9-11 nm, mean length of the nanotubes $1.2 \mu \mathrm{m}$, and purity higher than $90 \%$. During melt extrusion ventilation was kept on to remove trapped air in blends. After pelletizing, the nanocomposite granules were compression moulded into $4 \mathrm{~mm}$ thick plates using a hydraulic press at $180^{\circ} \mathrm{C}$ during 2 min for rheological experiments. For mechanical testing, nanocomposite granules were injectionmoulded [using KraussMaffei KM80-160E injection moulding machine] into standard test specimen for tensile, impact and flexural test. The barrel temperature ranged $205-220^{\circ} \mathrm{C}$ and the mould temperature was kept at $25^{\circ} \mathrm{C}$. The holding pressure and screw rotational speed were $300 \mathrm{bar}$ and $100 \mathrm{rpm}$, respectively. The nanocomposites of PP/MWNT and $2 \mathrm{wt} \%$ PP-g-MA filled PP/MWNT were fabricated by varying nanotube content starting from 
1 to $5 \mathrm{wt} \%$. As a reference, neat PP was also similarly processed for rheological and mechanical studies.

\subsection{Morphological characterization}

Scanning electron microscopic images were taken on the cryofactured surfaces of nanocomposite. A thin layer of carbon was sputter deposited onto the sample. Electron microscopy imaging of the nanocomposite was performed under high vacuum with a Hitachi S-4300SE/N SEM instrument operating at $5 \mathrm{kV}$.

\subsection{Rheological measurements}

Oscillatory shear measurements were carried out using an Advanced Rheometrics Expansion System (ARES) equipped with a cone and plate geometry device $(25 \mathrm{~mm}$ diameter $0.1 \mathrm{rad}$ cone angle and $0.4554 \mathrm{~mm}$ gap) at $180^{\circ} \mathrm{C}$. Frequency sweeps with an angular velocity between 0.1 and $100 \mathrm{rad} / \mathrm{s}$ were performed in the linear viscoelastic regime at strain of $5 \%$. Samples were left to equilibrate for $5 \mathrm{~min}$ utes prior to measurement.

\subsection{Mechanical characterization}

Mechanical performance of all compounded materials was evaluated from injection moulded specimens at $25^{\circ} \mathrm{C}$. Tensile properties of the moulded dogbone specimens were measured using an Instron machine at a crosshead rate of $20 \mathrm{~mm} / \mathrm{min}$ according to the ISO 527 standard. The tensile strength and modulus could be directly obtained from the stress-strain curves. Flexural properties of the nanocomposites were determined on the same tensile machine by three point bending tests as per ISO 178 standard at a thickness to span length ratio of 1:16 and at cross head displacement rate of $2 \mathrm{~mm} / \mathrm{min}$. Charpy notched and un-notched impact tests were carried out as per ISO 179-1 standard on a Zwick impact pendulum. All the reported values were calculated as averages over five specimens for each composition.

\section{Results and discussion}

\subsection{Investigation of the optimum PP-g-MA concentration}

In order to evaluate the optimum concentration of PP-g-MA in PP/MWNT nanocomposites, tensile behaviour of PP/MWNT nanocomposites was investigated at various PP-g-MA loadings by keeping $2 \mathrm{wt} \%$ of MWNTs constant. It is very important to keep in mind that too low PP-g-MA content would not be able to enhance the compatibility enough, while too high maleic anhydride content may lead to deterioration of the nanocomposite properties because of the poor mechanical properties of low molecular weight PP-g-MA. Therefore, it is necessary to investigate the optimum concentration of PP-g-MA. Figure 1 shows the tensile properties of PP/MWNT composites without PP-g$\mathrm{MA}$ and at different weight percentages of PP-gMA [i.e. 1, 2 and $3 \mathrm{wt} \%$ ]. It is clear from the results that tensile properties of the composites with PP-gMA has shown better tensile properties than PP/MWNT alone and the properties tend to increase with increase in PP-g-MA content up to $2 \mathrm{wt} \%$ and decrease for $3 \mathrm{wt} \%$ PP-g-MA nanocomposites. This is due to the fact that, PP-g-MA of low molecular weight and high MA content led to better interaction with nanotubes and PP matrix promoting the dispersion of nanotubes. However, the addition of lower molecular weight PP-g-MA had a negative effect on mechanical properties of the PP/MWNT nanocomposites. Therefore, there exists an optimum concentration of PP-g-MA for the pro-

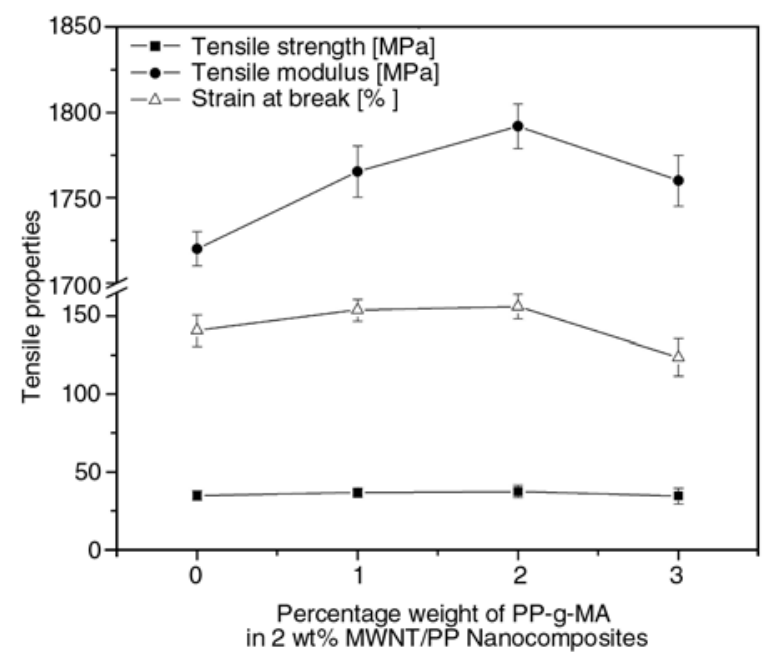

Figure 1. Effect of PP-g-MA content on tensile properties of PP/MWNT nanocomposites 
cessing of highly concentrated PP/MWNT masterbatches.

\subsection{Morphological characterization}

The efficiency of nanofillers in reinforcing the polymer matrix is primarily determined by the degree of its dispersion in the matrix. Therefore, morphological characterization is very important for the evaluation of the dispersion state of carbon nanotubes in the polymer matrix. In this study, dispersion of MWNTs in PP/MWNT and PP/MWNT/ PP-g-MA nanocomposites was examined by using SEM. The SEM images of composites containing shown in Figure 2. The selected SEM observations are representative of the dispersion of the carbon nanotubes in the PP matrix. For PP/MWNT nanocomposites (Figures $2 \mathrm{a}$ and $2 \mathrm{~b}$ ), a large amount of self-organized MWNTs bundles was observed on the cryofractured surface. This indicates that a sig-
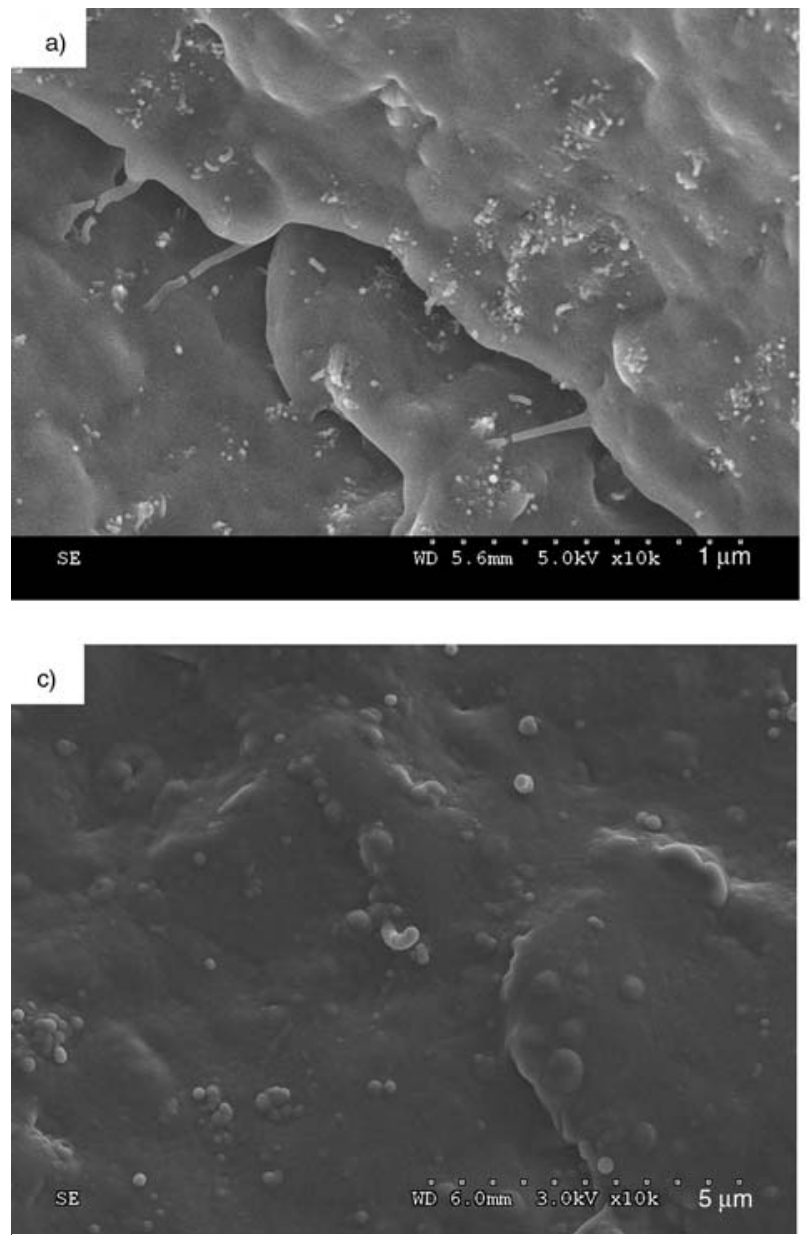
$3.0 \mathrm{wt} \%$ MWNTs with and without PP-g-MA are

nificant part of the nanotubes was dispersed as nanotubes aggregates due to the imperfect mixing of the masterbatch. But in the PP/MWNT/PP-g-MA composites (Figures $2 \mathrm{c}$ and $2 \mathrm{~d}$ ), reasonably uniform dispersion and good distribution of the MWNTs was observed with a small amount of aggregates. From these observations one can conclude that the added PP-g-MA promotes the dispersion of individual carbon nanotubes and limits the presence of nanotubes aggregates.

Moreover, in the case of the PP/MWNT/PP-g-MA composite (Figures $2 \mathrm{c}$ and $2 \mathrm{~d}$ ), the diameter of the MWNT has increased to about 150-200 nm, while in case of PP/MWNT diameter is about $30-40 \mathrm{~nm}$. The diameter of the nanotubes was determined from different SEM observations at different places for each composite so as to be representative of the average diameter. As the nanotube used for the different composites is the same, this increase in the diameter of the nanotubes is assumed to be due to wrapping of nanotubes by PP-g-MA thanks to
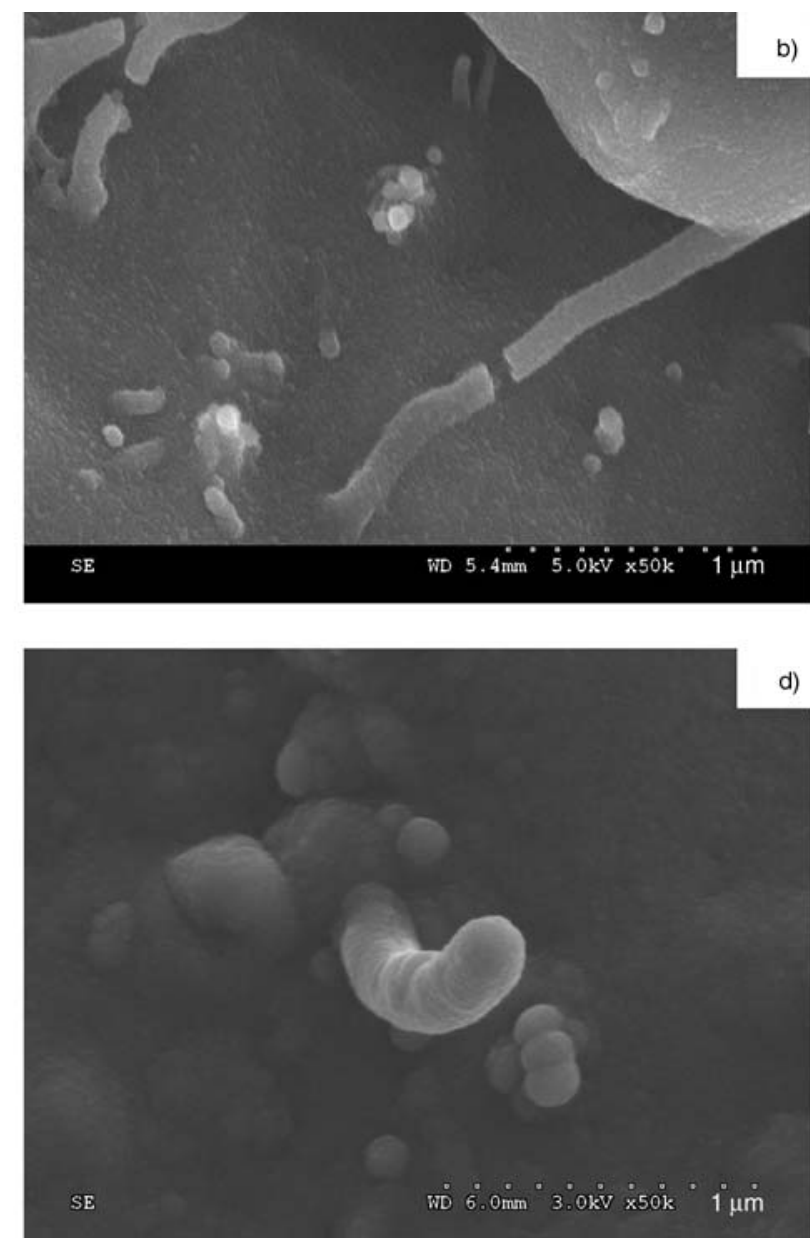

Figure 2. High and low resolution scanning electron micrographs of (a) and (b) PP/MWNT \& (c) and (d) PP/MWNT/PPg-MA melt mixed bulk samples 
hydrogen bonding. During extrusion process, due to specific interactions between the chemical groups of the carbon nanotube surface and grafted maleic anhydride groups, the PP-g-MA adsorbs onto the nanotubes giving a coating of a few nanometers thickness. Indeed, as shown by Lee et al. [22] hydrogen bonding between maleic anhydride and the surface-bound acid groups of the nanotube is possible, leading to the formation of a PP-g-MA coating onto the surface. After cryofracture, due to the adhesion of PP-g-MA on the nanotubes, this coating remains as observed by SEM (Figure 2d). In contrary, for PP/MWNT composites, no coating is observed and the measured diameter is in the range given by the CNT supplier. This tends to confirm the existence of the coating obtained via the addition of PP-g-MA during extrusion for PP/ MWNT/PP-g-MA composites. Further adhesion of PP-g-MA on the nanotube surfaces, modifies the wettability of the nanotubes and promotes the disentanglement in the PP matrix leading to a better dispersion during melt processing.

\subsection{Rheological characterization}

So as to support the SEM observations rheological measurements were carried out to obtain a qualitative evaluation of the nanotubes dispersion. Indeed, the rheological properties of the melt are sensitive to the structure, particle size, shape and surface properties of the dispersed phase and thus to the dispersion. Finally, it gives an overview of the dispersion of the nanotubes at the macroscopic level that is complementary with SEM observations. Moreover, it is important to evaluate the rheological behavior in order to understand the effect of the nanotubes on internal structures and processing properties of polymer/MWNT composites.

Figure 3 represents the frequency dependence of the storage modulus $\left(G^{\prime}\right)$ for the PP/MWNT and PP/MWNT/PP-g-MA nanocomposites measured at $180^{\circ} \mathrm{C}$. It is apparent that storage modulus of the nanocomposites increase with increase in MWNT content. In case of PP and $1 \mathrm{wt} \% \mathrm{PP} / \mathrm{MWNT}$ nanocomposites, at low frequencies PP chains are fully relaxed and exhibit typical homopolymer-like terminal behavior. However, when the nanotube loadings reaches $2 \mathrm{wt} \%$, this terminal behavior disappears and the dependence of $G^{\prime}$ on $\omega$ at low frequency is limited.

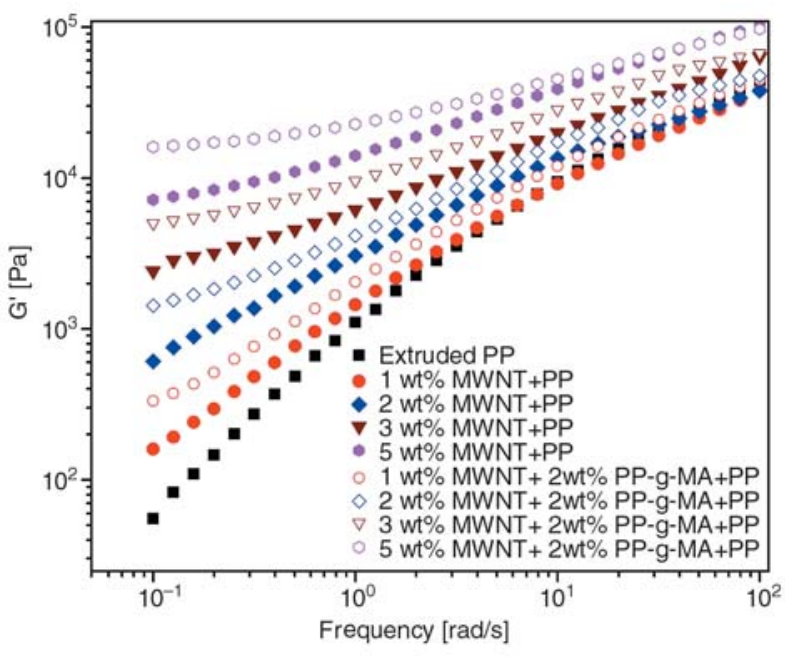

Figure 3. Storage modulus of PP/MWNT (filled symbols) and PP/MWNT/2 wt\% PP-g-MA (empty symbols) nanocomposites as a function of frequency at $180^{\circ} \mathrm{C}$

As shown in Figure 3, when the nanotube loading reaches $2 \mathrm{wt} \%, G^{\prime}$ becomes less frequency dependent at low frequencies, which indicates a transition from liquid-like to solid-like viscoelastic behavior [26]. This behaviour is characteristic of non-terminal frequency behaviour. This non terminal behaviour can be attributed to the formation of an interconnected nanotube network in the polymer (so-called percolation threshold). Therefore, the solid-like (or pseudo-solid-like) behavior can be attributed to the existence of frictional interactions between the highly anisotropic particles [27, 28]. When 2 wt\% of PP-g-MA is added to PP/MWNT nanocomposites, storage modulus of the composites was further increased. The rheological percolation threshold dropped from $2 \mathrm{wt} \%$ MWNTs (without PP-g-MA) to $1 \mathrm{wt} \%$ MWNTs when PP-gMA is added to PP/MWNT composites. The results indicate that PP-g-MA would allow developing favourable interaction between PP and MWNTs, and thus, better interconnected and percolated structure of MWNTs was produced in polypropylene matrix.

In order to better identify the rheological percolation threshold, the phase angle $(\delta)$ has been plotted against the frequency (Figures $4 \mathrm{a}$ and $4 \mathrm{~b}$ ). Indeed, due to the formation of an interconnected network of carbon nanotubes and thus to the transition from liquid-like to solid-like behaviour, $\tan \delta$ is independent of the frequency above the percolation threshold. Below the percolation threshold due to the liquid-like behaviour, the $\tan \delta$ increases at low 

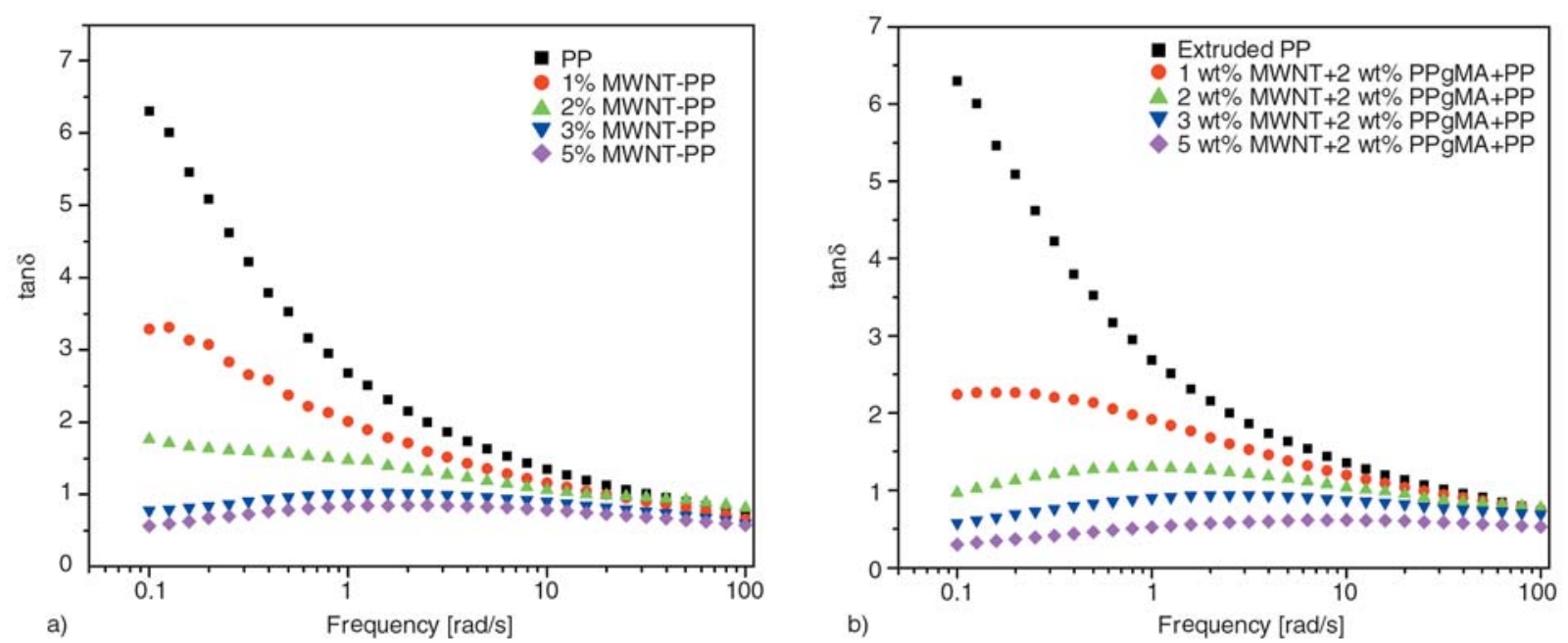

Figure 4. Phase angle $(\tan \delta)$ of PP/MWNT (a) and PP/MWNT/2 wt $\%$ PP-g-MA (b) nanocomposites as a function of frequency at $180^{\circ} \mathrm{C}$

frequency. Figures $4 \mathrm{a}$ and $4 \mathrm{~b}$ clearly indicate that the rheological percolation threshold for the PP/ MWNT composites is between 2 and $3 \mathrm{wt} \%$ nanotubes, whereas it is between 1 and $2 \mathrm{wt} \%$ for PP/MWNT/PP-g-MA composites. This decrease in percolation threshold concentration could be due to better dispersion of the nanotubes in the PP matrix thanks to PP-g-MA addition, in agreement with the SEM observations.

Logarithmic plot of $G^{\prime}$ versus $G^{\prime \prime}$ (Cole-Cole plot) is shown in Figure 5. Generally, Cole-Cole plot can be used as an effective method to explain structural changes occurring as a result of filler loading into a polymer matrix at a fixed temperature $[29,30]$. In case of PP/MWNT nanocomposites (Figure 5a), this plot deviates from that of the PP matrix when the nanotube content is $2 \mathrm{wt} \%$ in PP matrix. However, in case of PP/MWNT/PP-g-MA nanocompos-

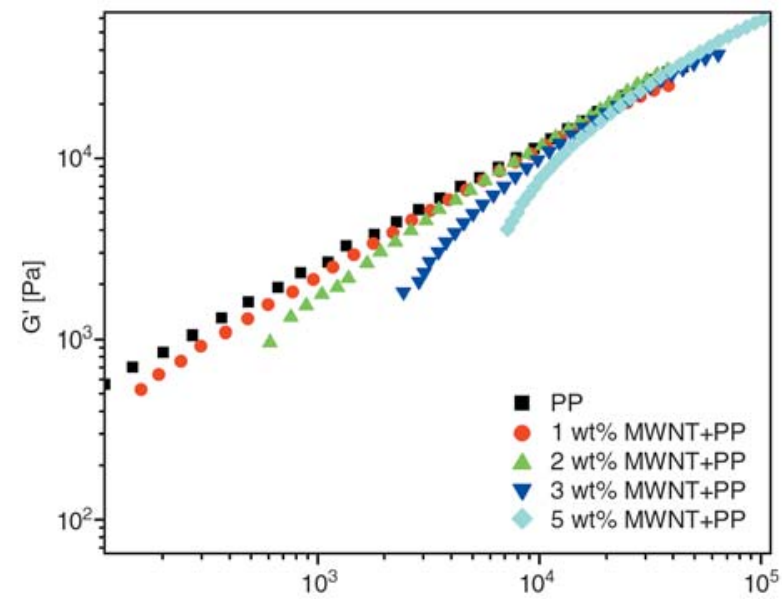

a) G" $[\mathrm{Pa}]$ ites (Figure 5b), the deviation from PP is already observed at $1 \mathrm{wt} \%$ nanotube. These observations indicate a structural change on the addition of nanotubes into polymer matrix. Further, these structural changes are more prominent in PP-g-MA bearing nanocomposites, showing a larger degree of deviation and thus a better dispersion. This is due to the fact that, addition of PP-g-MA into highly concentrated PP/MWNT masterbatches, increases the interfacial interaction between $\mathrm{PP}$ and MWNTs. The better wettability of the PP-g-MA coated nanotubes tends to favour the disentanglement of the bundles and promotes the dispersion of individual nanotubes limiting the remaining nanotubes aggregates. The increasing amount of welldispersed individual nanotubes thus allows to decrease the percolation threshold.

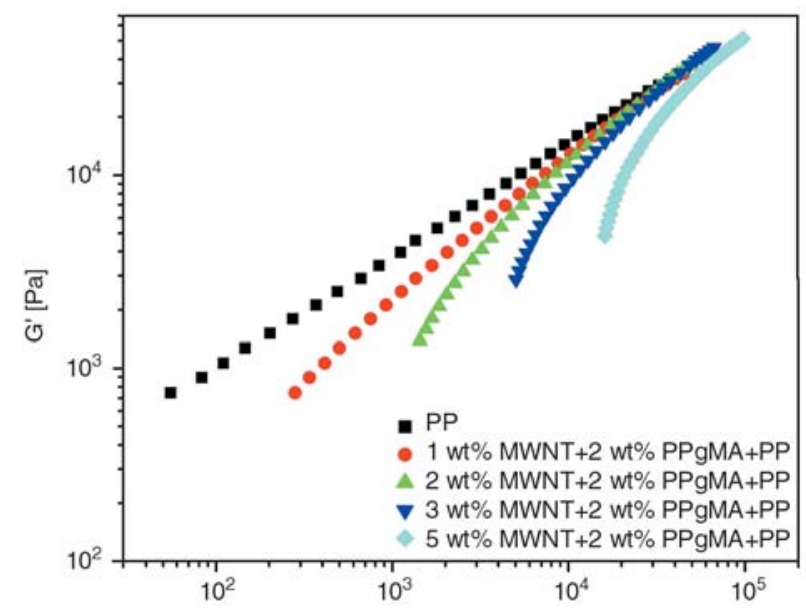

b) G" [Pa]

Figure 5. Cole-Cole plot for PP/MWNT (a) and PP/MWNT/2 wt\% PP-g-MA (b) nanocomposites 


\subsection{Mechanical characterization}

The variation of tensile strength, modulus and elongation at break with MWNTs content for the PP/MWNT and PP/MWNT/2 wt\% PP-g-MA composites are shown in Figure 6. It is obvious that Young's modulus (Figure 6a) and yield stress (Figure $6 \mathrm{~b}$ ) of PP/MWNT/PP-g-MA composites are higher than those of PP/MWNT composites, and tends to increase substantially with the MWNTs content in composites. It is now well-known and commonly admitted that the mechanical properties of polymer nanocomposites, especially the modulus, depend to a great extent on filler dispersion and interfacial interaction, and are increased only when good dispersion of the nanofiller and effective stress transfer at the polymer/filler interface are guaranteed. Indeed increasing the interfacial properties increases the volume of polymer chains influ-

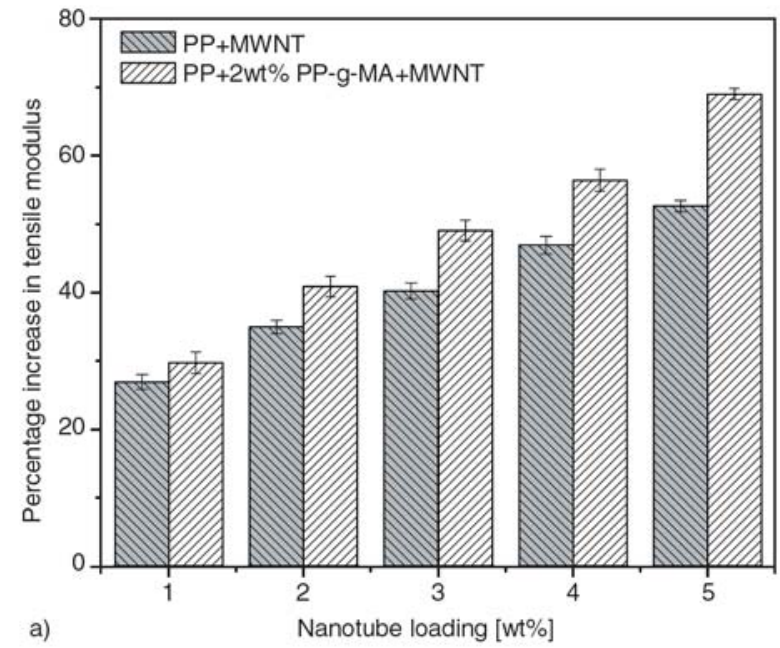

enced. The improvement of the dispersion also increases the average aspect ratio of the nanofillers in the polymer matrix and as a consequence tensile properties are improved as already reported [31] for clay platelets. Here apparently, MWNTs are able to reinforce the PP matrix due to their large aspect ratio combined with high mechanical properties. Incorporation of PP-g-MA into PP/MWNT, further increases the Young's modulus (Figure 6a) and yield stress (Figure $6 \mathrm{~b}$ ) of PP/MWNT/2 wt\% PP-gMA composites when compared to PP/MWNT nanocomposites. When PP-g-MA was added as compatibilizer, the tensile modulus of the composites treated with PP-g-MA increased on average $10 \%$ compared with the composite without PP-gMA. The effect of the PP-g-MA was even more pronounced in tensile yield stress (Figure 6b), where an average improvement of $35 \%$ was

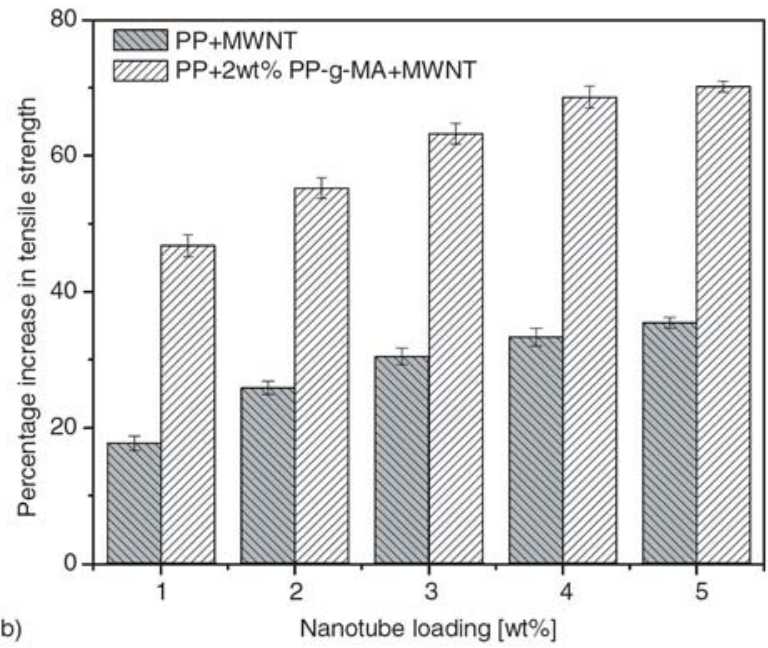

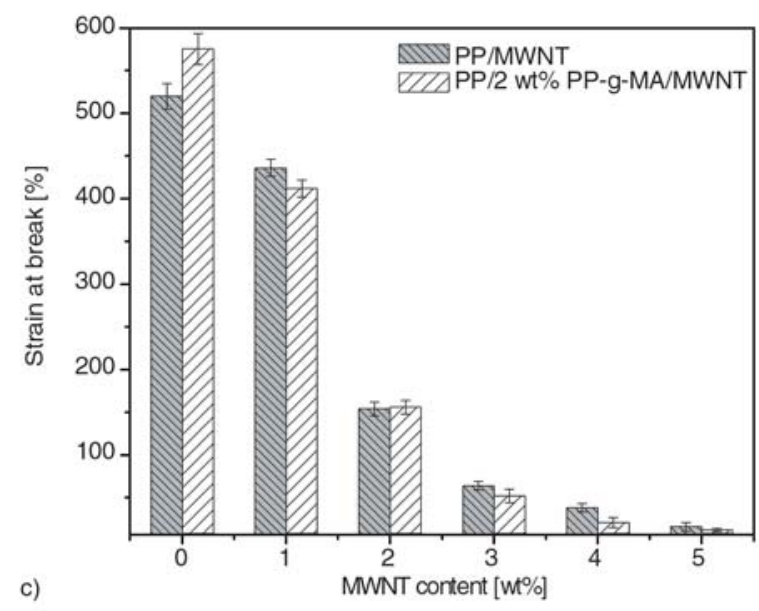

Figure 6. Percentage increase in tensile modulus with respect to PP and PP-g-MA as a function of MWNT content for the PP/MWNT and PP/MWNT/2 wt\% PP-g-MA composites (a). Percentage increase in tensile strength with respect to PP and PP-g-MA as a function of MWNT content for the PP/MWNT and PP/MWNT/2 wt\% PP-gMA composites (b). Variation of percentage strain at break with MWNT content for the PP/MWNT and PP/MWNT/2 wt\% PP-g-MA composites (c). 
observed. The increase of the modulus is due to the fact that PP-g-MA improves the dispersion of MWNTs in PP matrix and thus the aspect ratio and the carbon nanotubes/PP interface. On the other hand, PP-g-MA due to the wrapping of nanotubes modifies drastically the interface leading to the interfacial interaction enhancement and thus promotes a better stress transfer between MWNTs and PP matrix. The number and the size of the nanotubes aggregates is also reduced leading to a better tensile strength. In the case of PP/MWNT composites the aggregates act as stress concentrators easing the plastic deformation thus decreasing the yield stress. Finally, due to few aggregates and better interface, the yield stress is increased when PPg-MA is added. In spite of the better dispersion of MWNTs to PP matrix thanks to PP-g-MA, elongation at break decreases with increase in MWNT content (Figure 6c). This is probably due to the presence of few masterbatch aggregates.

The flexural strength and modulus of PP/MWNT and PP/MWNT/2 wt\% PP-g-MA nanocomposites are shown in Figure 7. It can be seen that the bending properties of PP/MWNT nanocomposites increased with increasing nanotube content up to a content of $2 \mathrm{wt} \%$ of MWNT, but decreases after $3 \mathrm{wt} \%$. Such a decrease in flexural properties at higher nanotube loading may probably be due to the fact that, the nanotubes formed a cluster or agglomerate among themselves, resulting in a filler-filler interaction and a poor interface between MWNTs and PP matrix. However, on addition of 2 wt\% PP-g-MA to PP/MWNT masterbatch, flexural properties of the composites are increased with

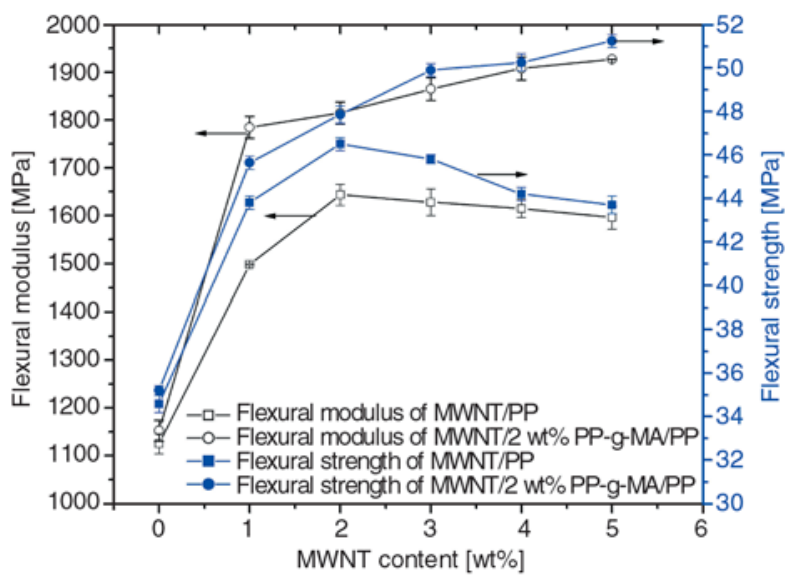

Figure 7. Flexural strength and modulus of PP/MWNT and PP/MWNT/2 wt\% PP-g-MA nanocomposites increase in nanotube content. This may be related to the more uniform dispersion of nanotubes in the PP matrix, leading to better interfacial properties. These results also indicate an effective usage of PPg-MA to assist the dispersion of nanotubes as supported by rheological measurements.

Finally, Figure 8 shows the impact properties of notched (Figure 8a) and un-notched (Figure 8b) samples of PP/MWNT and PP/MWNT/2 wt\% PPg-MA composites. Charpy impact strength of the notched specimens (Figure 8a) slightly increased as the MWNTs content increased up to $2 \mathrm{wt} \%$ for $\mathrm{PP} / \mathrm{MWNT}$ nanocomposites and up to $3 \mathrm{wt} \%$ in case of PP/MWNT/2 wt\% PP-g-MA nanocomposites. This increase is significantly larger for the samples with PP-g-MA. This is due to the fact that notched impact behavior is controlled to a greater extent by factors affecting the propagation of fracture initiated due to stress concentration at the notch tip. Lowering of impact energy at higher nanotube content is due to the presence few master-
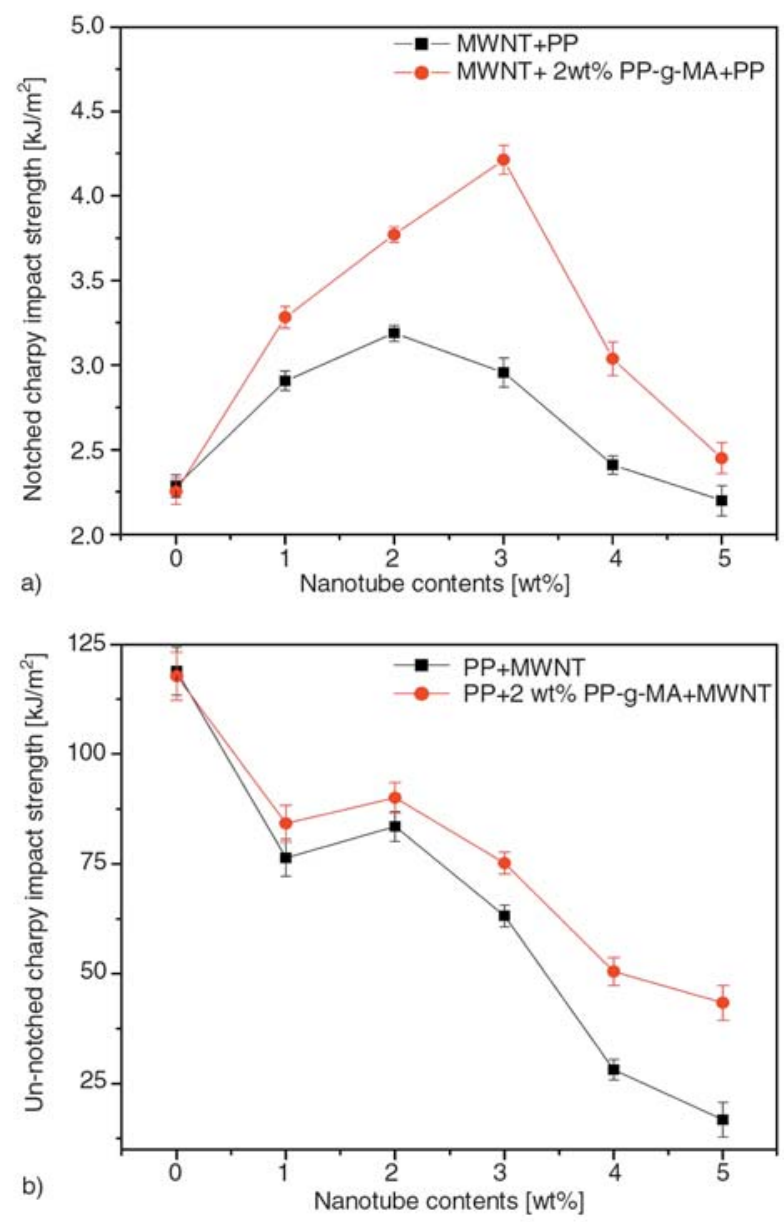

Figure 8. Impact properties PP/MWNT (a) and PP/ MWNT/2 wt\% PP-g-MA (b) nanocomposites 
batch aggregates in the composites. However, impact strength of the un-notched samples decreases with increase in MWNTs content in both the systems (Figure 8b). The decrease of impact energy in PP-g-MA bearing nanocomposites is nevertheless lower when compared to composites without PP-gMA. This is due to the effect of PP-g-MA during processing, leading to better dispersion of nanotubes in PP matrix. This observation is in contradiction with the results obtained for the elongation at break for which the composites based on PP-gMA are slightly more brittle. This apparent contradiction between effects of PP-g-MA on the elongation at break and on the impact strength values may be due to the differences in the deformation rate involved in the two types of experiments. Tensile testing involves high deformation, while impact strength is determined by local cracking. The detrimental effects of low molecular PP-g-MA are more pronounced when measuring elongation at break at low rate.

\section{Conclusions}

Multi-wall carbon nanotubes (MWNTs) filled polypropylene (PP) nanocomposites were prepared by melt compounding via masterbatch dilution technique. Polypropylene grafted maleic anhydride (PP-g-MA) was added to promote the carbon nanotubes dispersion.

The morphological characterization based on scanning electron microscopy has confirmed that nanotubes are distributed uniformly indicating a good dispersion of nanotubes in the PP/PP-g-MA matrix compared to pure PP. Thus, the results indicated that PP-g-MA is an effective dispersing agent for MWNTs based masterbatches in PP/MWNT nanocomposites. It is assumed that PP-g-MA promotes the dispersion thanks the enhanced interaction between MWNTs and PP due to wrapping of PP-gMA on MWNTs.

The rheological characterization has shown that, when PP-g-MA is added to PP/ MWNT nanocomposites, dynamic moduli and viscosity further increase. Liquid to solid transition takes place at a MWNTs content just above $1 \mathrm{wt} \%$, indicating a percolated network structure in the material, which appears earlier than for PP/MWNT without PP-gMA (transition at about $2 \mathrm{wt} \%$ in that case).
The mechanical characterization has shown that both the tensile and bending moduli and strengths of the nanocomposites increase by the addition of nanotubes, and the addition of PP-g-MA further improves these properties. An issue, which needs to be further investigated, is however that tensile elongation at break decreases with the addition of PP-gMA. Regarding impact properties, the Charpy impact strength increases, passes through a maximum and then decreases for notched samples, whereas for un-notched samples, a decrease is observed with the addition of MWNTs due to the presence of nanotubes aggregates. Nevertheless, the addition of $2 \mathrm{wt} \%$ PP-g-MA to the composites tends to improve these impact properties.

In conclusion, it has been demonstrated that the use of PP-g-MA is a way to promote a better dispersion of carbon nanotubes in a PP matrix, and thus better mechanical properties and lower rheological percolation threshold. Moreover the possibility to coat the nanotubes with PP-g-MA directly in the extruder is a very promising way to promote, insitu during the extrusion, the disentanglement of the carbon nanotubes. This technique may be extend to other matrices by changing the type of compatibilizer.

Furthermore, these results reveal that diluting thermoplastics/MWNTs masterbatches to manufacture plastic parts is a very promising route. Such a route allows keeping the functional benefits of well-dispersed MWNTs, whereas limiting the handling difficulties in plastics processing industrial workshops.

\section{Acknowledgements}

The authors are grateful for the financial support granted by Conseil Regional, Nord-Pas-de-Calais, France. Thanks are also due to Nanocyl SA. (Belgium) for providing the MWNTs masterbatch.

\section{References}

[1] Valentino O., Sarno M., Rainone N. G., Nobile M. R., Ciambelli P., Neitzert H. C., Simon G. P.: Influence of the polymer structure and nanotube concentration on the conductivity and rheological properties of polyethylene/CNT composites. Physica E: Low-dimensional Systems and Nanostructures, 40, 2440-2445 (2008). 
[2] Bikiaris D., Vassiliou A., Chrissafis K., Paraskevopoulos K. M., Jannakoudakis A., Docoslis A.: Effect of acid-treated multi wall carbon nanotubes on the mechanical, permeability, thermal properties and thermo-oxidative stability of isotactic polypropylene. Polymer Degradation and Stability, 93, 952-967 (2008).

[3] Ganß M., Satapathy B. K., Thunga M., Weidisch R., Pötschke P., Jehnichen D.: Structural interpretations of deformation and fracture behavior of polypropylene/multi-walled carbon nanotube composites. Acta Materialia, 56, 2247-2261 (2008).

[4] Qian D., Dickey E. C., Andrews R., Rantell T.: Load transfer and deformation mechanisms in carbon nanotube-polystyrene composites. Applied Physics Letters, 76, 2868-2870 (2000).

[5] Jin L., Bower C., Zhou O.: Alignment of carbon nanotubes in a polymer matrix by mechanical stretching. Applied Physics Letters, 73, 1197-1199 (1998).

[6] Zeng J., Saltysiak B., Johnson W. S., Schiraldi D. A., Kumar S.: Processing and properties of poly(methyl methacrylate)/carbon nano fiber composites. Composites Part B: Engineering, 35, 173-178 (2004).

[7] Li C., Pang X-J., Yu Z-L.: Study on polycarbonate/ multi walled carbon nanotubes composites produced by melt processing. Materials Science and Engineering: A, 457, 287-291 (2007).

[8] Chae H. G., Sreekumar T. V., Uchida T., Kumar S.: A comparison of reinforcement efficiency of various types of carbon nanotubes in polyacrylonitrile fiber. Polymer, 46, 10925-10935 (2005).

[9] Zhang X., Liu T., Sreekumar T. V., Kumar S., Hu X., Smith K.: Gel spinning of PVA/SWNT composite fiber. Polymer, 45, 8801-8807 (2004).

[10] McIntosh D., Khabashesku V. N., Barrera E. V.: Nano-composite fiber systems processed from fluorinated single-walled carbon nanotubes and polypropylene matrix. Chemistry of Materials, 18, 4561-4569 (2006).

[11] McIntosh D., Khabashesku V. N., Barrera E. V.: Benzoyl peroxide initiated in-situ functionalization, processing and mechanical properties of single-walled carbon nanotube-polypropylene composite fibers. Journal of Physical Chemistry C, 111, 1592-1600 (2007).

[12] Zhou Z., Wang S., Lu L., Zhang Y., Zhang Y.: Isothermal crystallization kinetics of polypropylene with silane functionalized multi-walled carbon nanotubes. Journal of Polymer Science Part B: Polymer Physics, 45, 1616-1624 (2007).

[13] Vaisman L., Marom G., Wagner H. D.: Dispersions of surface-modified carbon nanotubes in water-soluble and water-insoluble polymers. Advanced Functional Materials, 16, 357-363 (2006).

[14] López Manchado M. A., Valentini L., Biagiotti J., Kenny J. M.: Thermal and mechanical properties of single-walled carbon nanotubes-polypropylene composites prepared by melt processing. Carbon, 43, 1499-1505 (2005).
[15] Xiao Y., Zhang X., Cao W., Wang K., Tan H., Zhang $\mathrm{Q}$.: Dispersion and mechanical properties of polypropylene/multiwall carbon nanotubes composites obtained via dynamic packing injection molding. Journal of Applied Polymer Science, 104, 1880-1886 (2007).

[16] Shi D., Lian J., He P., Wang L. M., Xiao F., Yang L., Schultz M. J., Mast D. B.: Plasma coating of carbon nanofibers for enhanced dispersion and interfacial bonding in polymer composites. Applied Physics Letters, 83, 5301-5303 (2003).

[17] Kelarakis A., Yoon K., Sics I., Somani R. H., Chen X., Hsiao B. S.: Shear-induced orientation and structure development in isotactic polypropylene melt containing modified carbon nanofibers. Journal of Macromolecular Science, Part B: Physics, 45, 247-261 (2006).

[18] Zhou X., Xie X., Zeng F., Li R. K-Y., Mai Y-W.: Properties of polypropylene/carbon nanotube composites compatibilized by maleic anhydride grafted SEBS. Key Engineering Materials, 312, 223-228 (2006).

[19] Tjong S. C., Liang G. D., Bao S. P.: Electrical behavior of polypropylene/multiwalled carbon nanotube nanocomposites with low percolation threshold. Scripta Materialia, 57, 461-464 (2007).

[20] Jiang X., Bin Y., Kikyotani N., Matsuo M.: Thermal, electrical and mechanical properties of ultra-high molecular weight polypropylene and carbon filler composites. Polymer Journal, 38, 419-431 (2006).

[21] Lee S. H., Cho E. N. R., Jeon S. H., Youn J. R.: Rheological and electrical properties of polypropylene composites containing functionalized multi-walled carbon nanotubes and compatibilizers. Carbon, 45, 2810-2822 (2007).

[22] Lee G-W., Jagannathan S., Chae H. G., Minus M. L., Kumar S.: Carbon nanotube dispersion and exfoliation in polypropylene and structure and properties of the resulting composites. Polymer, 49, 1831-1840 (2008).

[23] Prashantha K., Soulestin J., Lacrampe M. F., Krawczak P., Dupin G., Claes M.: Compounding of masterbatch-based multi-walled carbon nanotube filled polypropylene nanocomposites: Assessment of rheological and mechanical properties. in 'Proceedings of the $8^{\text {th }}$ International Seminar on Experiment Techniques and Design in Composites Materials. Cagliari, Italy' 38-40 (2007).

[24] Ganß M., Satapathy B. K., Thunga M., Weidisch R., Pötschke P., Jehnichen D.: Structural interpretations of deformation and fracture behaviour of polypropylene/multi-walled carbon nanotube composites. Acta Materialia, 56, 2247-2261 (2008).

[25] Bao S. P., Tjong S. S.: Mechanical behaviours of polypropylene/carbon nanotube nanocomposites: The effects of loading rate and temperature. Material Science and Engineering A, 485, 508-516 (2007).

[26] Seo M-K., Park S-J.: Electrical resistivity and rheological behaviors of carbon nanotubes-filled polypropylene composites. Chemical Physics Letters, 395, 44 48 (2004). 
[27] Pötschke P., Fornes T. D., Paul D. R.: Rheological behavior of multiwalled carbon nanotube/polycarbonate composites. Polymer, 43, 3247-3255 (2002).

[28] Abdel-Goad M., Pötschke P., Zhou D., Mark J. E., Heinrich G. J.: Preparation and rheological characterization of polymer nanocomposites based on expanded graphite. Journal of Macromolecular Science, Part A: Pure and Applied Chemistry, 44, 591-598 (2007).

[29] Choi J. S., Lim S. T., Choi H. J., Pozsgay A., Százdi L., Pukánszky B.: Viscoelastic properties of exfoliated polyamide-6/layered silicate nanocomposite. Journal of Material Science, 41, 1843-1846 (2006).
[30] Chae D. W., Lee K. H., Kim B. C.: Rheological properties of ferrite nanocomposites based on nylon-66. Journal of Polymer Science Part B: Polymer Physics, 44, 371-377 (2006).

[31] Kojima Y., Usuki A., Kawasumi M., Okada A., Kurauchi T., Kamigaito O.: Synthesis of nylon 6-clay hybrid by montmorillonite intercalated with $\varepsilon$-caprolactam. Journal of Polymer Science Part A: Polymer Chemistry, 31, 983-986 (1993). 